\title{
Essentielle convergence de pensées entre Écologie, Interprétation, Muséologie et Patrimoine
}

\section{Annette Viel}

\section{OpenEdition}

\section{Journals}

Édition électronique

URL : https://journals.openedition.org/ere/3706

DOI : $10.4000 /$ ere.3706

ISSN : 2561-2271

Éditeur

Centr'ERE

Référence électronique

Annette Viel, «Essentielle convergence de pensées entre Écologie, Interprétation, Muséologie et Patrimoine », Éducation relative à l'environnement [En ligne], Volume 15 - 1 | 2019, mis en ligne le 29 octobre 2019, consulté le 15 octobre 2021. URL : http://journals.openedition.org/ere/3706 ; DOI : https://doi.org/10.4000/ere.3706

Ce document a été généré automatiquement le 15 octobre 2021

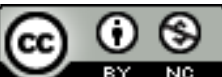

La revue Éducation relative à l'environnement est mise à disposition selon les termes de la Licence Creative Commons Attribution - Pas d'Utilisation Commerciale 4.0 International. 


\title{
Essentielle convergence de pensées entre Écologie, Interprétation, Muséologie et Patrimoine
}

\author{
Annette Viel
}

« Nous en sommes au préliminaire dans la constitution d'un paradigme de complexité luimême nécessaire à la constitution d'une paradigmatologie. Il s'agit non de la tâche individuelle d'un penseur, mais de l'œuvre historique d'une convergence de pensées. »

Edgard Morin

1 Au cours des deux derniers siècles, les champs d'investigation et d'action propres à l'écologie et à l'interprétation patrimoniale ont peu à peu pris en compte l'interaction dynamique qui caractérise l'ensemble des écosystèmes (Viel, 1995a, 2011a). Les enjeux qui ont marqué la genèse de ces deux domaines ont imposé une vision disciplinaire renouvelée au sein de laquelle émerge cette convergence de pensées décrite par le sociologue Edgard Morin $(1991,1992)$. Cette dynamique évolutive a guidé nos sphères de recherches et d'interventions, nous incitant à mieux baliser la nature des orientations conceptuelles inhérentes à la mise en valeur des multiples lieux patrimoniaux auxquels nous avons été associés tant au Canada qu'en Europe et Afrique. En effet, dans le cadre de nos travaux, nous tenions à ce que les projets développés soient en prise avec un territoire ouvert et dynamique, un territoire branché sur un développement dit durable et ce, d'autant que Parcs Canada a joint le ministère de l'Environnement au début des années quatre-vingt ${ }^{1}$.

Dès nos premières recherches portant sur l'origine de l'interprétation patrimoniale, nous avons constaté que le développement de l'interprétation entrait en écho avec l'écologie, invitant les acteurs du patrimoine à agir en interaction avec le territoire tel que le soutient l'écologie lors des premiers écrits définissant son champ d'investigation ${ }^{2}$. Au cours de la seconde moitié du XXe siècle, l'éducation relative à 
l'environnement emboîte le pas à la philosophique écologique et interprétative incitant les citoyens à être des acteurs conscients de l'essentielle protection des territoires particulièrement lorsque la préoccupation pour le devenir planétaire devient d'intérêt public (Villemagne, 2010 ; Sauvé, 2017). Cette philosophie rejoignait alors celle qui nous servait de balises pour l'élaboration de nos projets particulièrement concernant le volet éducatif inhérent à la conception de l'expérience interprétative (Viel 1990, 1991,1992, 1994, 2005a).

3 Notre propos se structure autour de quatre axes de réflexion qui sont apparus au fil de nos recherches guidant nos pratiques vers une approche pluridisciplinaire favorisant des passerelles entre les théories et les pratiques :

- L'interprétation : une incontournable assise de la création du réseau des parcs nationaux

- L'interrelation entre interprétation, écologie, et muséologie

- L'interprétation comme stratégie éducative

- Un patrimoine axé vers un développement citoyen

\section{L'interprétation, une incontournable assise de la création des parcs}

\section{Protéger le territoire en associant le citoyen}

Protecteurs de la nature, les premiers interprètes organisent des visites guidées sur les sites qu'ils veulent protéger, incitant les visiteurs à prendre conscience de leur soudaine fragilité au regard d'un développement territorial qui ne serait respectueux de la conservation des écosystèmes qui signent leur unicité (Raffin, 2005). Dès lors, l'interprétation commencera à se structurer et à s'implanter en force au cœur de l'Amérique ${ }^{3}$. Déjà, par les activités qu'ils offrent aux touristes désireux de s'évader des villes industrielles, les Catlin, Langford, Muir reconnaissent que l'éducation aux valeurs dont sont dépositaires ces lieux, se fera par une interprétation qui sache toucher le cœur tout autant que la raison. Cette nouvelle approche de sensibilisation patrimoniale prend le nom d'interprétation prenant appui sur la nécessité de développer une approche d'éducation informelle basée sur l'expérience globale (Viel, 1994, 2017). Lors de l'expédition menant à la création du parc Yellowstone, Nathaniel P. Langford écrivait : " pendant que vous regardez et vous émerveillez, vous constatez qu'il vous faut également comprendre à fond le milieu et y croire. $»^{4}$. Quelques années plus tard, John Muir, dont les écrits influenceront la création dans les montagnes de la Sierre Nevada de la Californie, du Parc de Yosemite, parlera ainsi de l'interprétation: «J'interprèterai les rochers, apprendrai le langage des inondations, des tempêtes et des avalanches. Je me familiariserai avec les glaciers et les jardins sauvages et me rapprocherai le plus possible du cœur du monde. $»^{5}$ Ces mots empreints de poésie, expriment de manière sensible, ce que signifie, pour les premiers grands interprètes, cette démarche interprétative qui repose sur une expérience holistique et spirituelle de la nature (Langford, 1905).

5 Un des premiers défenseurs de ces lieux d'exception fut George Catlin, avocat et artiste peintre, à qui plusieurs historiens reconnaissent la paternité de l'idée de parc (Mackintosh, 1999). Revenant d'un voyage effectué en 1832 dans l'Ouest californien, l'artiste défendit la nécessité de protéger ces espaces uniques où une beauté d'exception émerge d'une nature sauvage encore préservée du développement urbain. 
L'artiste insista alors sur la nécessité de protéger le territoire "par une grande politique de protection gouvernementale...dans un parc magnifique... ». Il développera au fil de ses écrits l'idée de la création d'espaces protégés. Nous citons de mémoire un extrait d'une lettre produite en 1832, dans laquelle Catlin écrivit : «Quel magnifique et palpitant spécimen à préserver et à maintenir pour l'Amérique, à la vue de ses citoyens distingués et du monde, dans les âges futurs! Le parc d'une nation, contenant homme et bête, dans toute la pureté et la fraîcheur de leur beauté naturelle. ${ }^{6}$

6 Yosemite en 1864 et Yellowstone en 1872 forment les deux maillons originaux du réseau des parcs d'Amérique! Le premier fut soustrait à toute forme d'exploitation grâce au décret initié par Abraham Lincoln et voté par le Congrès, ce qui en fit un parc régional car cet espace protégé relevait des autorités californiennes et n'accèdera au statut de parc national qu'en 1890 (Jacquin-Royot, 2002). Le 1er mars 1872, le Congrès américain crée le parc national Yellowstone protégeant ainsi plus de 8983 kilomètres carrés dont la nature fut jusqu'alors épargnée d'un développement humain ; un " parc public ou lieu de loisir pour le bénéfice et la jouissance des gens. $»^{7}$ Le bénéfice associé à la délectation et au plaisir constitue donc une des pierres angulaires de la loi qui régit tous les parcs nationaux. Deux années plus tard, le président américain Thomas Jefferson note : "Si ce n'est par sa culture, la nature de l'Amérique au moins doit faire l'admiration du monde.» (Conan, 1993). Les parcs des États-Unis forment un réseau reconnu et qui, bon an, mal an, continue d'attirer un grand nombre de touristes. En 2017, on y a dénombré plus de 331 millions de visites ${ }^{8}$.

7 Le Canada suivra de près le mouvement de conservation des parcs américains par la création de Banff, en 1885, au cœur des montagnes Rocheuses, alors qu'on est à y construire le chemin de fer qui permettra de réunir le pays de l'est à l'ouest (Lothian, 1997). Les objectifs initiaux de ce premier parc canadien étaient de préserver ses eaux sulfureuses d'un développement tous azimuts assurant ainsi la pérennité des lieux (Campbell, 2011, 2012). Le premier maillon du réseau canadien des parcs nationaux est marqué à la fois par la notion de tourisme, notion intégrée au texte fondateur, ainsi que par la recherche d'un équilibre entre conservation et mise en valeur. "Par conséquent, la transformation d'une région de plusieurs centaines de kilomètres carrés en réserve pour une utilisation à l'état naturel constituait vraiment une innovation dans la gestion des ressources.»(Lothian, 1997, p. 22) ${ }^{9}$. Le réseau canadien des parcs demeure également fort populaire auprès de la population et des touristes puisqu'on dénombre plus de 27 millions de visiteurs pour l'année $2017-2018^{10}$.

\section{Une première école de guides-interprètes fondée par Mills en 1916}

8 On ne peut parler de l'histoire du développement de l'interprétation sans citer Enos Mills, un des guides-interprètes américains les plus connus du début du XXe siècle, pour qui l'interprétation avait une portée tout autant "spirituelle qu'informative" (Mills, 1920-1990). Doté d'une faible santé, Mills se tourna dès son plus jeune âge vers l'observation de la nature et, un peu plus tard, subit l'influence de John Muir rencontré sur la plage de San Francisco en 1889. Ce dernier le prit sous son aile l'encourageant notamment à écrire. Ardent défenseur de la conservation de la nature, on lui reconnaît la création du parc national Rocky Mountains, situé au Colorado, territoire où il organise des excursions de 1899 à sa mort. 
9 Mills possède une insatiable curiosité pour tout ce qui touche à la nature. Il développe rapidement une approche analytique au regard $d u$ travail d'interprète particulièrement concernant le contact et les réactions avec les visiteurs. Il tente ainsi constamment de porter un regard critique de manière à améliorer les techniques d'interprétation qu'il développe. Mills croit en sa mission comme guide-interprète. Passionné par son travail, soucieux de créer un lieu d'enseignement afin de partager ce qu'il a acquis au fil de son expérience, il fonde en 1916, une première école pour les guides interprètes. Mills voyait trois éléments structurant son approche : la nature sauvage (wilderness), le guide et le visiteur nommé invité. Il lui importait de former des jeunes à sa philosophie de protection de ces territoires d'exception car il reconnaissait à ce qu'il nomme la "nature sauvage » une capacité à offrir une zone de sécurité et de protection face aux rigueurs du monde prétendument civilisé11.

10 Mills publie plusieurs centaines d'articles et plus d'une quinzaine de livres dont le plus connu demeure The Adventures of Nature Guide and Essays in Interpretation, sorti en 1920 et réédité en 1990 dans lequel il écrit : «La nature nous guide tel que nous la voyons, c'est spirituel avant d'être informatif. »(Mills, 1920, p. 6, traduction libre). Cet ouvrage a marqué l'histoire de l'interprétation pratiquée en Amérique bien avant Tilden. L'interprétation constitue une philosophie d'enseignement et d'action in-situ dont les prémisses théoriques ont été structurées par Enos Mills, lui-même initié par John Muir. Ce dernier mettait l'accent interprétatif sur le territoire alors que la force de Mills fut d'enrichir cette première approche par l'importance d'y associer implicitement le visiteur. Ces enseignements sont un repère significatif pour tout ce qui suivra par la suite dans le domaine de la protection consciente des environnements, domaine qui prend appui sur l'importance de sensibiliser le citoyen aux réalités écosystémiques : une forme d'éducation relative à l'environnement.

\section{L'interrelation entre écologie, interprétation et muséologie ${ }^{12}$}

\section{XIXe siècle : naissance de l'interprétation et de l'écologie}

L'interprétation du patrimoine naturel apparait, aux États-Unis, dès la première moitié du XIXe siècle, alors que plusieurs individus prennent conscience que la montée en flèche de l'industrialisation menace l'exceptionnelle beauté naturelle de ces régions encore épargnées de l'urbanisation (Brunelli, 2013). Ce plaidoyer pour la protection de la nature sera soutenu par des citoyens américains dont certains sont de souche européenne (Specq, 2008, p. 30). Les premiers défenseurs de la valeur esthétique et spirituelle de ces parcelles de territoires, la plupart situés dans l'Ouest américain, appuient leur revendication sur l'idée de protéger la pureté d'un paysage "sauvage » en opposition à un paysage marqué par l'intervention humaine. Ces gardiens patrimoniaux sont préoccupés par les conséquences environnementales qui découlent des aménagements orientés vers le développement économique urbain et la croissance d'un tourisme à la recherche de lieux naturels remarquables. Nous sommes cependant loin d'une vision et approche globales fondées sur la considération intrinsèque de l'ensemble des écosystèmes impliqués dans la dynamique structurelle d'un territoire. Par exemple, la création des premiers parcs tels Yellowstone aux États-Unis en 1872 ou Banff au Canada en 1885, en feront les frais dans l'enclave de protection initiale. En 
effet, la zone de protection de ces parcs repose davantage sur les réalités paysagères idylliques du territoire protégé que sur une vision d'ensemble prenant appui sur la dynamique des écosystèmes du territoire auxquels ils appartiennent. Le respect de l'intégrité écologique ne faisait pas encore partie de l'approche de protection et de mise en valeur des parcs et réserves telle qu'on la retrouve aujourd'hui (Woodley, 2010, p. 151-160).

ailleurs, au milieu du XIXe, soit en 1866, la définition de l'écologie apparaît en Europe grâce, entre autres, aux recherches menées par le médecin, biologiste et philosophe allemand Ernst Haeckel. Ce scientifique prend appui sur les recherches initiées par des collègues naturalistes de renom dont les travaux de l'allemand Alexander Von Humboldt, de l'anglais Charles Darwin et du français Jean-Baptiste de Lamarck. Son champ d'investigation se tourne résolument vers les interrelations entre toutes formes de vie terrestre. Il relie les mots maison et économie créant ainsi un nouveau terme à partir des origines grecques : oikos pour habitat/maison et logos pour discours/domaine. L'écologie désigne la «science de l'économie, des habitudes, des relations mutuelles des organismes.» (Drouin, 1992, p.54). En ouvrant sur la compréhension des relations mutuelles de ces organismes, l'écologie nous convie à recourir à une approche pluridisciplinaire pour assurer une meilleure intégration des multiples facettes auxquelles nous convoquent les lieux « nature/culture » (Viel, 1994).

\section{Années 70 et 80 : environnement, développement durable et approches muséales pluridisciplinaires}

Il faudra cependant attendre de nombreuses années pour que, notamment, de ce côté-ci de l'Atlantique, en Amérique du Nord, l'écologie devienne partie prenante de la compréhension des territoires particulièrement lorsqu'il s'agira de la conservation de la nature dans le respect de son intégrité écologique. Les ministères dédiés à l'environnement ne feront leur apparition qu'au cours des années $70 \mathrm{du}$ siècle passé tout comme, d'ailleurs, l'écomuséologie défendue par l'ethnologue français GeorgeHenri Rivière et surtout, Hugues de Varine qui est le créateur de ce concept (Varine, 1978). La Conférence mondiale de l'environnement tenue à Stockholm en $1972^{13}$, la Charte de Belgrade de 1975, la commission Brundtland de 1987, autant de rencontres internationales importantes qui prôneront la nécessité de voir de manière globale les interventions des sociétés, invitant celles-ci à œuvrer à un développement durable respectueux des ressources (Bergandi et Blandin, 2012). Après la rencontre de Stockholm, d'autres sommets de la terre seront mis sur pied afin de mieux orchestrer des programmes globaux de veille environnementale (Viel, 1994, 1995b, 2011). La montée en flèche de la préoccupation écologique fait peu à peu son entrée dans la sphère patrimoniale et muséale (Viel, 1989, 1991, 1994, 1995a).

$\mathrm{Au}$ cours de ces décennies, l'écomuséologie se développe: les muséologues transposeront les fonctions muséales au territoire en interaction avec un patrimoine in-situ, là où usages et objets prennent racines. Peu à peu, les pratiques muséales traditionnelles s'enrichiront, notamment par la prise en compte de la dimension immatérielle (Schiuma, 2011, p. 2). Par ailleurs, au fil de ces années, l'éducation relative à l'environnement se structure, invitant citoyens et sociétés à considérer l'ensemble des actions et gestes de manière à assurer un développement durable à notre planète terre (La Branche-Mulot, 2010). 
15 En 1970, Parcs Canada ${ }^{14}$, qui avait son siège social dans la capitale canadienne Ottawa, décentralise une partie de la gestion de ses sites, donnant alors naissance à la région de Québec. Une équipe jeune et dynamique développera une approche originale respectueuse des directions mises de l'avant par le bureau central. Ainsi prendra forme une approche interprétative originale qui se développe sur la base d'une alliance entre science et créativité, une approche qui marquera le champ d'action muséologique (Viel, 1991). Dès les débuts des années 80 , alors que Parcs Canada intègre définitivement le ministère de l'Environnement, de nouvelles passerelles se feront entre les objectifs du nouveau ministère et ceux poursuivis par une organisation déjà centenaire. La prise de conscience de l'importance de conserver le territoire dans un souci de développement durable deviendra un des objectifs de toute mise en valeur (Viel et Nivart, 2005b). L'interprétation gagnera en force tant auprès des guides qui œuvrent en prise directe avec les usagers des parcs que des professionnels qui ont en charge de créer l'expérience de visite pour ces lieux d'exception. Le réseau québécois s'enrichira de nombreux parcs et lieux historiques; les centres d'interprétation se multiplieront et l'approche thématique privilégiée permettra de renouveler la tradition muséologique.

\section{L'interprétation comme stratégie éducative}

\section{Interpréter : « nouvelle forme d'éducation »}

16 Près de cent ans se seront écoulés depuis la création des premiers parcs lorsque Freeman Tilden écrira Interpreting our heritage. Ce livre deviendra la référence en matière interprétative pour bien des générations. Tilden est avant tout un littéraire qui, lorsqu'il commence à s'intéresser à l'univers des parcs, a déjà produit livres de fiction et pièces de théâtre. Pour lui, l'interprétation constitue «la révélation de la vérité plus générale qui se cache derrière tout énoncé de fait. » (Tilden, 1974, p. 25, traduction Parcs Canada). Fortement encouragé, dès le début des années 50, par Newton B. Drury, directeur des parcs nationaux, à découvrir l'univers des parcs, Tilden publia un premier ouvrage en 1951 intitulé "The National Parks: What They Mean to You and Me ", un titre annonciateur des propos de Interpreting our heritage, qui paraîtra en 1957. D'autres titres de Tilden tels The State Parks, Following the Frontier with F. Jay Haynes : Pioneer Photographer of the Old West (1964) et The Fifth Essence, an Invitation to Share in Our Eternal Heritage (1968) vont dans le même sens mettant l'accent sur l'importance d'aborder l'interprétation en alliant cœur et raison! Lors de ces nombreux discours, Tilden référait souvent aux civilisations européennes afin d'étayer ses dires et donner à ce qu'il observait comme pratique interprétative une autre dimension : l'âme des choses.

17 L'interprétation doit chercher à toucher le visiteur et à l'interpeller à partir de ses propres expériences comme en témoigne un des six principes écrits par l'ancien journaliste: "Toute interprétation d'un paysage, d'une exposition ou d'un récit qui n'en appelle pas d'une façon ou d'une autre à un trait de la personnalité ou de l'expérience du visiteur est stérile.» (Tilden, 1957). Il reprendra sensiblement la philosophie du président américain Thomas Jefferson au regard de l'importance de l'innovation américaine en ce qui concerne la création des parcs. Il affirme que l'interprétation constitue une "nouvelle forme d'éducation basée sur une entreprise systématique de protection et d'utilisation du patrimoine national. L'ampleur de cette 
activité n'a pas d'équivalent dans les vieilles nations ni dans les autres époques.» (Tilden, 1957, p. ?).

18 La valeur de l'ensemble de l'œuvre de Freeman Tilden repose principalement sur le travail de synthèse que l'écrivain fit à partir de son observation de la pratique interprétative sur le terrain et de son expérience comme créateur artistique. Ses écrits firent école: Tilden fut la référence pour des milliers d'interprètes qui tentaient de comprendre et de mettre en pratique l'interprétation. L'auteur ne formulait pas d'approche méthodologique relative à la mise en valeur des lieux et de leur interprétation. Son livre présentait une esquisse de quelques principes fort généraux agrémentés d'un récit journalistique. Sa vision s'orientait davantage du côté artistique et humaniste qui, selon ses propos, caractérise l'univers de l'interprète.

Comme l'a exprimé l'écossais Don Albridge, l'interprétation c'est «l'art d'expliquer la place de l'homme dans l'environnement, de façon à augmenter, chez le visiteur ou dans le public, la conscience de l'environnement et à éveiller le désir de contribuer à sa conservation. » (Aldridge, 1975, p. 12). En 1998, Larry Beck et Ted Cable, deux anciens interprètes devenus universitaires, écrivent Interpretation for the 21st century, ouvrage qui revisite l'interprétation à la manière de Tilden: «un processus informel et inspirant conçu pour améliorer la compréhension, l'appréciation et la protection de notre héritage culturel et naturel » (Beck et Cable, 1998, p. xi, traduction libre). Fidèles à la philosophie de Tilden, Beck et Cable réactualisent ses six principes auxquels ils en ajoutent neuf autres. Leur livre ne constitue pas une histoire exhaustive de l'interprétation mais bien davantage une mise à jour des principes énoncés par Tilden en 1957. Comme les auteurs le soulignent : « Nous sommes bénis en tant qu'interprètes et c'est notre bénédiction de partager avec d'autres. » (p. 206, traduction libre)

\section{L'interprétation au cœur d'une sphère muséale en mutation}

20 Au début des années 70 , quelques années après que Parcs Canada a procédé à une première décentralisation administrative, les professionnels de l'interprétation œuvreront à stimuler le développement en force d'une interprétation patrimoniale novatrice. Ils créeront, entre autres, de nouvelles manières d'inciter les différents publics à vivre une expérience originale en adéquation avec de l'esprit des lieux, une expérience incitant à la prise de conscience de l'importance de la préservation des valeurs de ces lieux d'exception (Viel, 1990). L'interprétation n'est plus l'unique fait des guides interprètes mais également des infrastructures muséographiques mises en place pour faciliter la diversité de l'expérience des visiteurs de plus en plus nombreux. Lieux de synthèse qui renvoient les visiteurs au territoire à interpréter, les centres d'interprétation ${ }^{15}$ offrent des clés de compréhension de la raison d'être des parcs et réserves naturels ainsi que des lieux historiques. De plus, ces centres d'interprétation doivent proposer une vision élargie des connaissances scientifiques qui émergent de l'ensemble du site assurant ainsi un lien de sens avec le territoire auquel ils appartiennent. L'objectif est d'établir et de soutenir un dialogue entre la ressource et l'ensemble des citoyens, visiteurs et habitants. (Viel et Nivart, 2005b)

21 En 1984, devant l'essor de l'interprétation au Québec, l'Office de la langue française entreprend un vaste travail de cueillette de tous les mots utilisés par les professionnels de l'interprétation en milieu de la francophonie afin d'intégrer ces néologistes à la langue française. L'interprétation y est définie comme une « méthode de sensibilisation 
qui consiste à traduire, pour un public en situation, le sens profond d'une réalité et ses liens cachés avec l'être humain, en ayant recours à des moyens qui font d'abord appel à l'appréhension, c'est-à-dire qui mènent à une forme vécue et descriptive de la connaissance plutôt qu'à une forme rigoureusement rationnelle »(Cayer, 1984, p. 61).

$\mathrm{Au}$ cours des dernières décennies du XXe siècle, l'interprétation gagne peu à peu l'univers des musées : les parcs nationaux et les lieux historiques sont reconnus comme entité muséale (Viel, 1989). De part et d'autre, parcs nationaux, parcs régionaux, réserves naturelles, lieux historiques et musées développent de nouvelles relations entre lieux, objets et visiteurs conviant les citoyens à vivre une expérience polysémique alliant émotion et raison pour mieux apprécier ces lieux où sont conservés et mis en valeur ces " témoins matériels et immatériels de l'homme et de son environnement. »" Durant cette période, plusieurs muséologues internationaux questionnent les acquis et tentent une approche renouvelée pour cette science responsable du « sens des traces». Les débuts de l'histoire des musées montrent ces derniers comme de grands réservoirs cumulant les traces matérielles et immatérielles de l'homme et de son environnement tant immédiat que lointain. Le musée d'aujourd'hui témoigne d'une profonde mutation. Les professionnels de la muséologie ont renouvelé les approches traditionnelles tout en respectant ses assises et ce, dans les différents champs d'appropriation des multiples savoirs. En peu de temps, nous sommes passés d'une muséologie d'objets à une muséologie d'expériences au sein de laquelle l'interprétation y occupe une place prépondérante (Viel, 2005a).

\section{De Parcs Canada au Musée de la civilisation : place à une expérience diversifiée}

La population a été de plus en plus invitée à participer activement à ce courant de valorisation des traces patrimoniales. La progression des écomusées témoigne notamment de cette tendance muséale. Les expositions in-situ se sont multipliées et une nouvelle approche muséologique a gagné les territoires. On a reconnu d'emblée la nécessité d'ouvrir la science muséale à des réalités anthropologiques et sociétales globalisantes davantage enracinées dans l'environnement tant urbain que rural. Dès ses premiers balbutiements, au début des années 80, le Musée de la civilisation de Québec bénéficiera de ce nouvel élan muséologique qui prend force tout autant en Europe qu'en Amérique. Sa programmation thématique, son approche muséographique interactive, ses activités culturelles en écho avec la cité, ont attiré un public diversifié et nombreux. Fortement tributaire de la tradition d'interprétation développée dans les parcs et lieux historiques canadiens et américains, son approche muséologique repose sur une philosophie axée sur l'homme, acteur d'un monde en continuité et en devenir tout en mettant en scène des activités pluridisciplinaires (Arpin, 1994). L'objet de collection n'est plus le centre de la muséographie; il devient support d'une trame narrative qui a été structurée à partir de résultats de recherches scientifiques pluridisciplinaires conduisant à la création d'expositions et d'activités initiatrices de thématiques innovantes et modernes. Voilà l'émergence d'un changement important qui marquera la muséologie de la fin des années quatre-vingt où les musées de société opèrent une entrée signifiante au sein du monde muséal $!^{17}$ (Viel, 2003b) 


\section{Un patrimoine axé vers un développement citoyen : un exemple en Gaspésie} projet marqué par l'évolution sociale et environnementale des actions patrimoniales. Il s'agit de la mutation de la vieille usine de transformation du poisson de l'Anse à Beaufils, usine située en Gaspésie, qui constitue un exemple de la prise en main par les citoyens de leur patrimoine.

\section{En Gaspésie, plusieurs stratégies de patrimonialisation}

Comme bien des territoires porteurs de ressources, la mer n'est pas inépuisable ; au fil des décennies, la morue, jadis fleuron de l'économie gaspésienne montra des signes de rareté et les pêcheurs gaspésiens durent se tourner vers d'autres activités. Une des conséquences de ce changement économique fut, sans contredit, la fermeture de lieux consacrés à cette industrie. Plusieurs d'entre eux devinrent des lieux de mémoire dédiés à l'histoire d'autrefois et proposés comme produits touristiques. Ce fut le cas, par exemple, du Chafaud de Percé devenu le centre d'accueil et d'interprétation du parc national du Rocher Percé et de l'île Bonaventure; du site historique du Banc-dePêche de Paspébiac dont les huit bâtiments ont été transformés en lieux d'interprétation; de l'ancien village de Grande Grave dont une partie des bâtiments sert de support pour raconter, au cœur de Parc national Forillon, l'aventure des marchands jersiais et guernesiais ainsi que des pêcheurs et ouvriers associés à la production de la morue séchée, exportée à travers le monde au cours des siècles passés. Ces lieux furent mis en valeur au fil de leurs fermetures et de la nécessité de développer de nouveaux usages qui seraient davantage en phase avec une industrie axée sur le tourisme. Le Parc national Forillon fut ainsi créé en 1970 dans cette foulée de tentative de diversification économique assurant la protection d'un paysage grandiose accessible aux touristes. Pour la création du parc Forillon, tout comme celui de Percé-Île Bonaventure, on expropria ses habitants et on ferma les villages qui jalonnaient le territoire maintenant devenu «bien de la nation $»^{18}$. L'objectif poursuivi était alors de doter la région de sites touristiques d'exception afin de stimuler l'économie en phase de mutation (Viel, 1992 ; Babin, 2015).

Face à cette mutation économique, le havre de pêche de l'Anse à Beaufils, situé dans l'arrondissement de Percé, ne fut pas épargné : la fermeture de son ancienne usine de transformation de poisson, érigée depuis 1928, amena les citoyens à questionner sa possible mutation et surtout de sa réappropriation ${ }^{19}$. Mais comment ? Car à quoi bon transformer cette usine en musée ou centre d'interprétation alors que d'autres lieux situés dans la région occupaient déjà ce rôle ? Comment alors doter la vieille usine d'une vocation novatrice tout en respectant sa valeur mémorielle? C'est alors que quarante-neuf citoyens se réunirent et optèrent d'acquérir le lieu afin de le transformer en coopérative culturelle. Ainsi naquit, en 1998, la Vieille Usine qui, au fil des ans, devint un centre culturel populaire et diversifié, véritable lieu de rencontre entre le citoyen gaspésien et le touriste. Magnifiquement bien situé aux abords de l'Atlantique, jouxtant une plage réputée pour la beauté de ses agates, ce lieu offre les infrastructures essentielles pour les pêcheurs contemporains, les croisiéristes et les plaisanciers dans une marina de qualité. Des sentiers jalonnent la rivière adjacente au 
port proposant des excursions au cœur d'un territoire où la beauté de la nature ne se dément pas pour qui s'y aventure.

\section{Un lieu de création et d'interprétation, tributaire des expériences locales}

L'ancienne usine, classée bien patrimonial, a conservé son enveloppe extérieure d'autrefois tout en transformant ses espaces intérieurs au service de sa nouvelle vocation culturelle. On y trouve un bistro pourvu d'une terrasse où il fait bon manger les produits du terroir qui y sont apprêtés tout en se laissant aller à découvrir divers groupes de musiciens d'ici comme d'ailleurs lorsqu'ils y déploient leur talent. À ce bistro s'ajoutent une salle d'exposition, une boutique, des ateliers pour enfants, des artistes-artisans à l'œuvre, une salle de spectacle et un studio professionnel où musiciens et chanteurs peuvent enregistrer un produit de qualité comme dans les grandes villes. "En d'autres termes, partager le patrimoine gaspésien et valoriser la création artistique constituent notre raison d'être. Bref, La Vieille Usine est un lieu de création et d'interprétation ouvert sept (7) jours/semaine. C'est un véritable lieu de rencontre qui démocratise les arts et revitalise le patrimoine $! »^{20}$

Voilà donc un projet novateur fort populaire qui, tout en respectant la valeur historique du lieu, explore des avenues culturelles diversifiées et inspirantes pour l'ensemble des citoyens conviés à y vivre une expérience culturelle durable. Ainsi, par exemple, en 2014, les citoyens-acteurs qui ont investi dans la réhabilitation du lieu ont eu le plaisir de retrouver leur mise de fond. Tout un exploit et une grande satisfaction sociale dans un lieu dont l'offre culturelle fait événement à la fois pour l'habitant et le touriste tout en veillant à l'intégrité du territoire et de sa mémoire passée, présente et à venir! Un lieu dont l'expérience mise sur une diversité d'approches culturelles tout en protégeant la valeur de ses ressources patrimoniales naturelles et culturelles. Un lieu miroir de l'essentielle réappropriation citoyenne de ce début XXIe.

\section{Conclusion : une muséodiversité au cœur de territoires citoyens}

Les quatre points que nous avons abordés prennent appui sur ces convergences de pensées et de sens qui ont soutenu nos recherches et actions: convergences entre écologie, interprétation, muséologie et patrimoine (Viel, 1992, 1994, 2011, 2016). Ces quatre domaines trouvent particulièrement écho dans des lieux de conservation et de mise en valeur bien ancrés dans le territoire (Viel, 2016). Le milieu de l'environnement a imposé, au fil des dernières décennies, la prise en compte de la biodiversité, celui de la muséologie et de la patrimonialisation offre une muséodiversité, reflet d'une culture vivante en prise avec ses traces et leur signifiance. Une muséodiversité dont les formes d'expression se multiplient, intégrant l'incontournable sensibilisation éducative à l'environnement! Rappelons que depuis la fin du siècle dernier, la culture constitue le quatrième pilier du développement durable au sein duquel la muséodiversité trouve tout son sens. (Viel, 2011)

Les musées, tout comme les autres lieux de mémoire et d'interprétation nature/ culture, doivent être partie prenante du mouvement de prise de conscience des 
incertitudes environnementales et de la nécessité d'entretenir des liens étroits entre société et environnement. Ces lieux constituent des espaces de vie et d'interprétations diversifiées qui participent à la continuité des cultures prenant appui sur la nature des territoires qui signent leur identité. Il importe que les lieux/objets matériels et immatériels jouent pleinement leur rôle d'acteurs territoriaux et ce, à part entière, au sein des sociétés auxquelles ils demeurent redevables. Leur programmation doit s'articuler sur les diverses facettes de ces parcelles identitaires dont les lieux demeurent garants. Ce souffle de vitalité ne saurait se passer d'une réelle réappropriation citoyenne initiant ainsi de nouveaux ancrages sociétaux porteurs de pérennité.

\section{BIBLIOGRAPHIE}

Pennyfather, K et Aldridge, D. (1975). Guide to Countryside Interpretation. Edinburgh : Her Majesty's Stationery Office

Arpin, R. (1994). Mission, concept et orientations. Québec : Publication du Musée de la civilisation

Babin, A. (2015) L'expropriation du territoire de Forillon, Les décisions politiques au détriment des citoyens. Québec : Les Presses de l’Université Laval

Bergandi, D. et Blandin, P., (2012). De la protection de la nature au développement durable : genèse d'un oxymore éthique et politique Revue d'histoire des sciences, 65, 103-142

Beck, L. et T. T. Cable. (1998). Interpretation for the $21^{\text {st }}$ Century: Fifteen Guiding Principles for Interpreting Nature and Culture. Champaign (Illinois): Sagamore Publishing

Brunelli, Martha, (2013). From nature guiding to nature interpretation in the United States (1872-1920). The origins of the professional practice of heritage interpretation: between protection and education. Dans Caroli, D. et Pomante, L. (Eds.), International Bibliography of History of Education and Children's Literature (p. 399-428). Macerata: Edizioni Università di Macerata Catlin, G. (1876). The North American indians with letters and notes (2 volumes). Londres: Chatto et Windus

Campbell, C. E. (2011). Pragmatism and poetry: National parks and the story of Canada. RCC Perspectives , no. 4, Big Country, Big Issues: Canada's Environment, Culture, and History, 101-111. Récupéré sur https://www.jstor.org/stable/10.2307/26240322

Campbell, C. E. (2012) A century of Parks Canada, 1911-2011. Calgary: University of Calgary Press, Canadian History and Environment Series. Récupéré sur https://press.ucalgary.ca/books/ 9781552385265/

Conan, M. (1993). La nature, la religion et l'identité américaine. Dans D. Bourg (dir.), Les sentiments de la nature (p. 175-195), Paris : La Découverte

Cayer, M. et al, (1984). Interprétation du patrimoine. Québec : Office de la Langue française, collection Néologie en marche, no 38-39 
Agence Parcs Canada (2000). Intacts pour les générations futures? Protection de l'intégrité écologique par les parcs nationaux du Canada. (Vol. I « Le temps d'agir »; vol. II « Une nouvelle orientation pour les parcs nationaux du Canada ». Ottawa : Rapport de la Commission sur l'intégrité écologique des parcs nationaux du Canada.

Drouin, J-M. (1992). L'écologie : généalogie d'une discipline. Dans Theys, J. et Kalaora, B. (dir.), La terre outragée - Les expert sont formels (p. 54-65). Paris : Autrement, collection « Sciences et sociétés »

Giordan, A. (1998). Apprendre. Paris : Éditions Belin, collection « Débats ».

Jacquin, P. et Royot, D. (2002). Go West ! Histoire de l'Ouest américain d'hier à aujourd'hui. Paris : Flammarion.

La Branche, S. et Milot, N., (2010) Enseigner les Sciences Sociales de l'environnement, Un manuel multidisciplinaire. Villeneuve d'Ascq : Presses universitaires du Septentrion, collection Environnement et société

Langford, N.P. (1905, réédition 1972). The Discovery of Yellowstone Park; Diary of the Washburn Expedition to the Yellowstone and Firehole Rivers in the Year 1870. Lincoln: University of Nebraska Press

Lothian,W. F. (1987). Petite histoire des parcs nationaux du Canada. Ottawa : Environnement Canada Parcs

Mackintosh, B. (1999). The National Park Service: A brief history. United States : National Park Service History Electronic Library. Récupéré sur http://npshistory.com/publications/ brief_history/index.htm

Morin, E. (2014). La Méthode (Tome 4) : Les Idées. Leur habitat, leur vie, leurs mœurs, leur organisation. Paris : Editions Le Seuil, collection Points (édition originale 1991)

Morin, E. (1992), La Méthode (Tome 3) : La Connaissance de la connaissance. Paris : Editions du Seuil. Mills, E. (1920, reedition 1990). The Adventures of a Nature Guide and Essays on Interpretation, Friendship. Wisconsin : New Past Press

Raffin, J-P. (2005), De la protection de la nature à la gouvernance de la biodiversité. Écologie \& Politique 2005/1, $\mathrm{n}^{\circ}$ 30, 97-109.

Sauvé L., Orellana I, Villemane C. et Barder B., (2017). Éducation, Environnement, Écocitoyenneté : Repères contemporains. Sainte-Foy : Presse de l'Université du Québec

Specq, F. (2008). Henry D. Thoreau et la naissance de l'idée de parc national. Ecologie \& politique, $2008 / 1, n^{\circ} 36,29-40$

Tilden, F. (1957 ; 1967). Interpreting Our Heritage. Chapel Hill: The University of North Carolina Press, édition révisée

Tilden, F. (1974). L'interprétation de notre patrimoine. Gatineau : Publication de Parcs Canada, référence E 1758 T5761967

Tilden, F. (1964). Following the Frontier with F. Jay Haynes: Pioneer Photographer of the Old West. New York: Alfred A. Knopf

Tilden, F. (1968). The fifth essence: An invitation to share in our eternal heritage. Rockville: National Park Trust Fund Board

De Varine, H. (1978, 1992). L'écomusée . Dans Desvallées, A. (dir.), Vagues : une anthologie de la nouvelle muséologie, volume. 1. Lyon : Presses Universitaire de Lyon 
Viel, A. (1989). Quand le lieu devient objet. Dans B. Schiele (dir.), Faire voir, faire savoir : la muséologie scientifique au présent (p. 73-82). Québec : Publication du Musée de la civilisation

Viel, A. (1990), La muséologie à l'heure écologique- entre le respect du public et la raison d'être d'un lieu patrimonial, collaboration Louise Boucher. Revue Musées, SMQ. vol. 12. No 3, 39-42

Viel, A. (1991) Parcs et lieux sur la voie muséale, Une approche écologique. Cap-aux-diamants, Revue d'histoire du Québec, 25, 40-47

Viel, A. (1992) Modalités d'accès : aujourd'hui pour demain. Les aléas de la rentabilité muséale. Logique symbolique ou logique économique. Actes des Troisièmes Rencontres Nationales des Musées, Musée et économie, juin 1991. Direction des Musées de France, 102-114

Viel, A. (1994). La veille environnementale, nouveau paradigme muséal. Dans Bravard, J-P. (dir.), Paysages de l'eau aux portes de la ville : mise en valeur écologique et intégration sociale (p. 174-185). Lyon : Presses Universitaire de Lyon. 174-185.

Viel, A. (1995a). La situation canadienne : Quand souffle l'esprit des lieux, Patrimoine naturel Patrimoine culturel. Paris : École du Patrimoine. 213-229.

Viel, A. (1995b). La Biosphère, lieu d'irrigation des savoirs. Revue Écodécision, 17-25.

Viel, A. (2003a). Quand le musée vit au rythme de la cité. Sens et contresens de « l'esprit des lieux ». Dans Younès C. (dir.), Art et philosophie, ville et architecture (221-235). Paris : Éditions La Découverte, collection Armillaire

Viel, A. (2003b). Pour une écologie culturelle. Culture \& Musées, nº 1, 139-149.

Viel, A. (2005a). L'objet dans tous ses états, Mot-Motion-Musée. Dans Mariaux P.A. (dir.), L'objet de la Muséologie (p. 51-81). Neuchâtel : Institut d'histoire de l'art et de Muséologie.

Viel, A. et Nicart, A. (2005b). Parcs sous tension. Culture \& Musées, n 5, 135-156. Récupéré sur : https://www.persee.fr/doc/pumus_1766-2923_2005_num_5_1_1217

Viel, A. (2011a). Une muséodiversité au cœur d'un développement dit « durable ». Dans Porceda A. et Chaumier S. (dir.), Musées et développement durable (p. 317-333). Paris : La Documentation française, collection « Musées-Mondes »

Viel, A. (2011b). Patrimoine et développement durable : un incontournable rapprochement. Dans Dubois, A-L. (coord.), Guide-Ane n 2 - Patrimoine et développement durable (p. 3-18). Cran-Gevrier : Éditions du Réseau Empreintes

Viel, A. (2016). Créativité et territoire(s) en mouvement : créer pour mieux vivre et habiter la pluralité territoriale. Revue Ethnologies, 38 (1-2), 213-236

Villemagne C. (2010). Regard historique sur le développement de l'éducation relative à l'environnement. Dans La Branche S. et Milot N. (dir.), Enseigner les sciences sociales de l'environnement (p. 17-30). Villeneuve d'Ascq: Presses Universitaires du Septentrion White, F. (2006). Essential Muir: A Selection of John Muir's Best Writings. Berkeley (Californie): Heyday Books.

Woodley, S. (2010). Ecological Integrity and Canada's National Parks. The George Wright Forum, vol. 27 (2), 151-160. Récupéré sur : www.jstor.org/stable/43598147 


\section{NOTES}

1. Nous avons œuvré au sein de la mise en valeur patrimoniale durant plusieurs années dont une bonne partie au sein de Parcs Canada. Par exemple, en 1991, nous avons orchestré les orientations conceptuelles suivantes : les lieux historiques nationaux de la Grosse île et de Fort Lennox, le parc marin Saguenay-Saint-Laurent, le musée de la Corse et celui de la Biosphère, premier musée de l'environnement au Canada.

2. Écologie : «science de l'économie, des habitudes, des relations mutuelles des organismes.» définition donnée par le médecin biologiste darwiniste allemand Ernst Haeckel. (cité dans Drouin, J-M., 1992, p. 8).

3. Les Parcs nationaux américains ont mis en ligne un grand nombre de documents d'archives: http://www.cr.nps.gov/history/index.htm

4. Tiré d'un dossier de formation des guides interprètes de Parcs Canada que nous avons maintes fois consultés. Malheureusement, nous n'avons pu retrouver ce dossier disparu depuis le démantèlement de l'ancien bureau régional de Québec en 2012.

5. Nous citons les mots de Muir à partir des notes que nous avions prises lors de nos recherches alors que nous oeuvrions à la réalisation d'un premier sentier d'interprétation en 1979 pour le parc Forillon.

6. Traduction Parcs Canada.

7. L'acte signé est maintenant numérisé : http://www.yellowstone-natl-park.com/history.html

8. https://www.statista.com/statistics/206820/number-of-visitors-to-national-park-servicesites-since-2008-2017 Date de consultation?

9. Notons cependant que le manque de connaissance de ce que constituait alors un écosystème et son intégrité écologique imposera une approche renouvelée à la fin du XXe. Une grande commission d'enquête sur l'intégrité écologique sera mise sur pied au début XXe au Canada; le rapport qui suivra imposera une approche renouvellée.

10. https://www.pc.gc.ca/fr/docs/pc/attend\#a1

11. Michael Mills Kiley, petit-fils de Mills, auteur de l'introduction de la réédition Adventures of a Nature Guide évoque longuement les motifs qui ont conduit Mills à développer l'interprétation.

12. Notre propos se centre sur le développement, en Amérique du nord, des parcs nationaux et de l'interprétation comme outil de mise en valeur. Notons que les premiers artisans de l'interprétation proviennent d'Europe et transposent avec eux les visions développées par les scientifiques et naturalistes menant des recherches sur différents territoires. Au fil du temps, ces recherches conduiront à la création de grandes institutions muséales tels les premiers muséums d'histoire naturelle.

13. La rencontre de Stocklom de 1972 constituera le premier Sommet de la terre.

14. Parcs Canada assure la gestion d'un important réseau de lieux sélectionnés pour leur valeur patrimoniale et donc culturelle au sein de l'histoire du pays : plus de 145 lieux historiques, 39 parcs naturels, 3 parcs marins, 8 canaux historiques de navigation et 26 rivières du patrimoine. Les ramifications du réseau s'étendent au niveau planétaire car treize d'entre eux, labellisés site du patrimoine mondial, sont placés sous l'égide de la Convention de l'UNESCO.

15. Lieu l'on se consacre principalement à la sensibilisation de la population en appliquant la méthode particulière qu'est l'interprétation » (histoire, nature, etc.).

16. Définition du musée selon ICOM : « Le musée est une institution permanente, sans but lucratif, au service de la société et de son développement, ouverte au public qui acquiert, conserve, étudie, expose et transmet le patrimoine matériel et immatériel de l'humanité et de son environnement à des fins d'études, d'éducation et de délectation. »

17. Le concept de musée de société apparait à la fin des années quatre-vingt; il sera officialisé lors du colloque Musées et Sociétés organisé à Mulhouse, en France, en 1991. 
18. Malheureusement l'expropriation des habitants de ces lieux demeure une entache à l'histoire de la création de nombreux parcs nationaux dont Forillon. Parcs Canada a d'ailleurs reconnu son erreur et des actes de réconciliation ont été mis sur pied au cours des dernières années.

19. Pour étoffer notre propos, nous avons, au cours de la dernière décennies, effectuées des recherches in-situ et rencontrés plusieurs des citoyens impliqués dans ce processus depuis ses premiers balbutiements dont Simon Poirier alors gérant du Café-Bistro et co-programmeur des spectacles.

20. http://lavieilleusine.qc.ca/web2/

\section{RÉSUMÉS}

À la lumière des recherches et des projets qui ont jalonné un ample parcours professionnel, déployé dans l'univers de la conservation et de la mise en valeur de lieux d'exception, l'auteure synthétise les éléments phares qui ont permis de créer des passerelles de sens entre les quatre domaines suivants : écologie, interprétation, muséologie et patrimoine. Le fil conducteur de l'article montre à quel point ces liens de sens ont reposé notamment sur le développement d'une dimension éducative inhérente à l'expérience proposée et vécue, que ce soit du côté de celui qui la définit ou de celui qui la vit. L'incontournable liant que constitue l'enracinement identitaire, inscrit dans des territoires maintenant en quête de nouveaux savoirs, ancrages, expériences et développements, demeure l'assise de toute action.

In the light of research and projects that have marked a rich professional career, a journey deployed in the world of conservation and the development of exceptional places, the author summarizes the key elements that have made it possible to create bridges of meaning between the four following areas : ecology, interpretation, museology and heritage. The main theme of the statements supported demonstrates the extent to which these links of meaning have rested, in particular on the development of educational components inherent to the experience proposed and lived either on the side of the one who defines it or the one who lives it. The unavoidable link that constitutes the rooting of identity, a rooted in territory now in search of new knowledge, anchors, experiences, and developments remains the basis of all actions.

\section{INDEX}

Keywords : interpretation, ecology, museology, heritage, spirit of places, citizen

Mots-clés : interprétation, écologie, muséologie, patrimoine, esprit des lieux, citoyen

\section{AUTEUR}

\section{ANNETTE VIEL}

Annette Viel, muséologue, consultante internationale, a développé une approche originale de la mise en valeur des lieux y intégrant la notion de respect de l'« esprit des lieux ». Elle a été professeur associée et invitée (2002-2007) au Muséum National d'Histoire Naturelle de Paris et à l'Université de Bourgogne (1999-2002). Elle a dirigé et collaboré à de multiples projets de création 
en muséologie et en patrimoine dans différents pays et continents. Elle prône une approche transdisciplinaire et diversifiée, n'hésitant pas à prendre des voies inédites alliant l'art aux projets développés. 